\title{
Pesquerías de medusa en la Costa Miskitu: desafíos para la gobernanza comunal después de la Ley 445 de Nicaragua
}

\author{
Jellyfish Fisheries on the Miskitu Coast: Challenges for \\ communal governance in Post-Law 445 Nicaragua
}

\author{
Matthew L. Fahrenbruch ${ }^{1}$ \\ Universidad de Kansas, USA.
}

\begin{abstract}
Resumen
La Costa Miskito es una región históricamente marginada de Nicaragua. Desde el período colonial, la Costa ha estado cultural y físicamente aislada del Pacífico, que es dominantemente mestiza. Durante años, la población 'costeña' ha exigido una mayor autonomía sobre el gobierno local. La aprobación de la Ley 445 ha transferido en el papel, algunas responsabilidades de gobernanza a los gobiernos comunales y territoriales, sin embargo, los desafíos siguen en pie. Me concentro aquí en los desafíos que enfrentan los gobiernos comunales cuando se trata de proyectos extractivos no tradicionales. Me enfoco específicamente en el desarrollo de la pesquería de medusas que comenzó en 2008. Mis resultados sugieren que los gobiernos comunales aún juegan un papel menor en el desarrollo de sus recursos naturales, carecen de apoyo y están en una desventaja significativa de poder y conocimiento cuando se trata de negociaciones con actores externos. Incluso después de la aprobación, las comunidades carecen de sistemas de contabilidad para los recursos extraídos y tienen pocas vías para capturar los ingresos de las actividades de extracción.
\end{abstract}

Palabras clave: Costa Miskitu, Nicaragua, Autonomía, Gobernanza multinivel, Marginalización

\section{Abstract}

The Miskito Coast is a historically marginalized region of Nicaragua. Since the colonial period, the Coast has been culturally and physically isolated from the dominant mestizo Pacific. For years, the 'costeño' population has demanded greater autonomy over local governance. The passage of Law

1 Candidato doctorado, Departamento de Geografía y Ciencias Atmosféricas, la Universidad de Kansas, M1fahrenbruch@gmail.com

Este artículo corresponde a la ponencia presentada en el 35th Conference of Latin American Geographers realizada en San José, Costa Rica del 20 al 22 de mayo del 2018. 
445 has devolved, on paper, some governance responsibilities to communal and territorial governments, however, challenges remain. I focus here on the challenges faced by communal governments when dealing with non-traditional externally generated extractive projects. I consider specifically the development of the jellyfish fishery that began in 2008. My results suggest that communal governments still play only a minor role in the development of their natural resources, lack advocacy, and are at a significant power and knowledge disadvantage when it comes to negotiations with external actors. Even after approval, communities lack systems of accounting for extracted resources and have few avenues for capturing proceeds from extraction activities.

Keywords: Miskitu Coast, Nicaragua, Autonomy, Multi-level governance, Marginalization

\section{Introducción}

Desde la época colonial, la Costa Miskitu² de Nicaragua (la Costa) ha experimentado varias economías de auge y depresión basadas en extracción de recursos que han impactado el ambiente, la economía y la cultura (Nietschmann 1979, Helms 1971, Offen 2004, Pineda 2006). A pesar de esto, los indígenas y afrodescendientes de la Costa han tenido históricamente poco control sobre la extracción industrial de recursos. La gobernanza de recursos se ha caracterizado por la dominación extranjera de los mercados, el control central del desarrollo, y la marginación de los derechos territoriales de los indígenas y afrodescendientes (Hale 1994; Nietschmann 1997; Vilas 1989; Baracco 2011).

Después de años de lucha por hacer realidad los derechos otorgados por la Ley de Autonomía de 1987, la Asamblea Nacional de Nicaragua (ANN) adoptó la Ley de las tierras comunales (Ley 445) en 2003 (ANN $2003)^{3}$. La ley facilitó la titulación de 23 territorios indígenas y afrodescendientes e instituyeron un sistema multi-nivel de gobernanza de los recursos naturales que garantiza a estas poblaciones una voz en los proyectos extractivos en sus tierras y aguas (Larson y Lewis 2012).

Entre 2008 y 2015, una nueva industria extractiva se desarrolló alrededor de las medusas. La medusa ha sido explotada en Asia oriental por lo menos 1.700 años como comida y medicina (Rudloe 1995; Hsieh et al., 2001). Históricamente, China fue el principal productor y consumidor de medusas. Sin embargo, la alta demanda y la sobreexplotación han resultado en precios más altos y la expansión de las pesquerías de

2 La costa de Miskitu en una región vernácula que se corresponde con la costa del Caribe y las regiones autónomas del norte y del sur. En los documentos oficiales, la Costa Miskitu se conoce como la Costa Caribe o la Costa Atlántica o como las Regiones Autónomas.

3 La ley de las tierras comunales es corto para, "Ley del Régimen de Propiedad Comunal de los Pueblos Indígenas y Comunidades Étnicas de las Regiones Autonomas de la Costa Atlántica y de los Ríos, Bocay, Coco, Indio y Maiz"

398 Revista Geográfica de América Central No 61E (3) Especial CLAG Este artículo está bajo una licencia e-ISSN 2215-2563 • Julio-diciembre 2018 • pp. 397-412 Atribución/Reconocimiento-NoComercialDoi: http://dx.doi.org/10.15359/rgac.61-3.20 
Matthew L. Fahrenbruch. Pesquerías de medusa en la Costa Miskitu: desafíos para la gobernanza comunal después de la Ley 445 de Nicaragua

medusas en mercados no tradicionales como Nicaragua (Brotz 2017; Fahrenbruch 2018).

El objetivo de este artículo es ofrecer una revisión preliminar de la pesquería de medusas dentro del contexto de la Ley 445 destacando varios desafíos que aún enfrentan los gobiernos comunales. De este modo, este artículo proporciona un estudio de caso empírico para contribuir a la literatura sobre gestión de los recursos naturales con la implementación de la Ley 445.

\section{Métodos}

Esta investigación se llevó a cabo durante 10 meses y tres visitas a la Costa entre 2015 y 2018 incluida una estancia de ocho meses entre agosto de 2017 y abril de 2018. Yo vivía principalmente en la Región Autónoma del Norte (RACCN) en la pequeña comunidad de Tuapi y en la capital de Puerto Cabezas (Bilwi). También visité las comunidades de Krukira y Bismuhna (RACCN) y Haulover en la Región Autónoma del Sur (RACCS) (Figura 1) ${ }^{4},{ }^{5}$.

4 Todas estas comunidades tenían plantas de procesamiento de medusas. También se informó que las plantas estaban ubicadas en Awastara, El Bluff y Monkey Point; sin embargo, no tuve la oportunidad de visitar estas comunidades.

5 Para convertirse en un producto comercializable, las medusas deben curarse con sal en un proceso que dura aproximadamente una semana. Este curado con sal se lleva a cabo en instalaciones especialmente construidas para su procesamiento. Para más información sobre el proceso general, ver Rudloe (1995) y específicamente para Nicaragua, ver Fahrenbruch $(2017,2018)$. 
Figura 1. Nicaragua and the Autonomous Regions (Miskitu Coast).

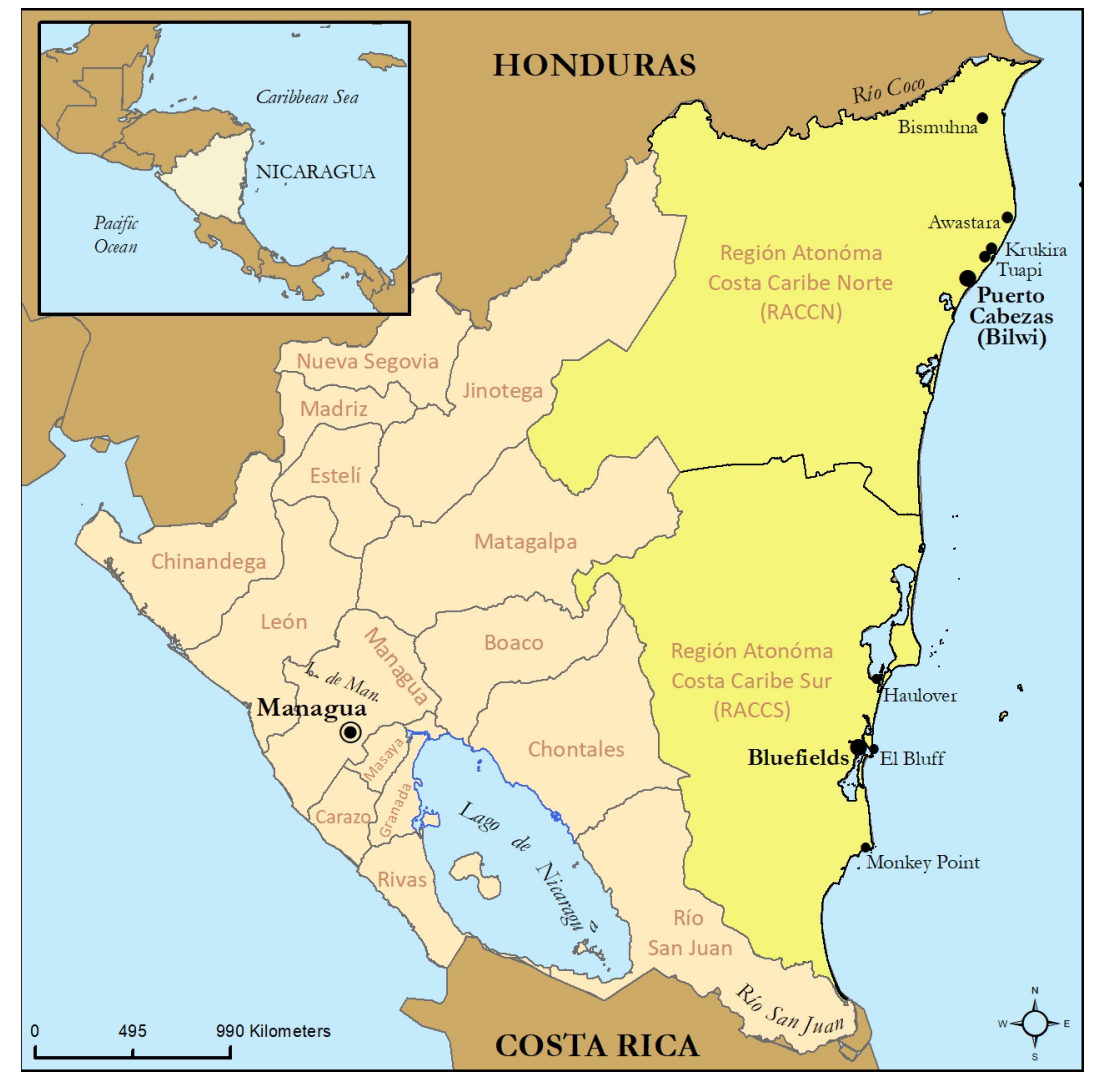

Fuente: Autor.

Los resultados discutidos aquí provienen de 30 entrevistas semiestructuradas e informales con funcionarios de la Oficina de La Secretaria de Recursos Naturales y el Ambiente (SERENA) en Bilwi, el Instituto Nicaragüense de la Pesca y Acuicultura (INPESCA) en Bilwi y Managua, de líderes, pescadores y trabajadores en las comunidades mencionadas, así como sesiones de escucha en Tuapi, Bismuhna y Haulover.

\section{La pesquería de medusas}

La primera planta para procesar medusas fue construida en 2008 en Tuapi (Figura 2). La especie objetivo fue la medusa bala de cañón (Stomolophus meleagris) (Figura 3) (Brotz et al. 2017). La planta fue construida por 
Matthew L. Fahrenbruch. Pesquerías de medusa en la Costa Miskitu: desafíos para la gobernanza comunal después de la Ley 445 de Nicaragua

Pesquera-Bilwi S.A. (PBSA), filial de Moon International, una empresa coreana-mexicana con sede en Sonora, México. Moon había procesado medusas del Golfo de California desde 2001, pero una mala temporada en 2007 los animó a desarrollar operaciones en Centroamérica (Fahrenbruch 2018).

Figura 2: La planta de medusas en Tuapi.

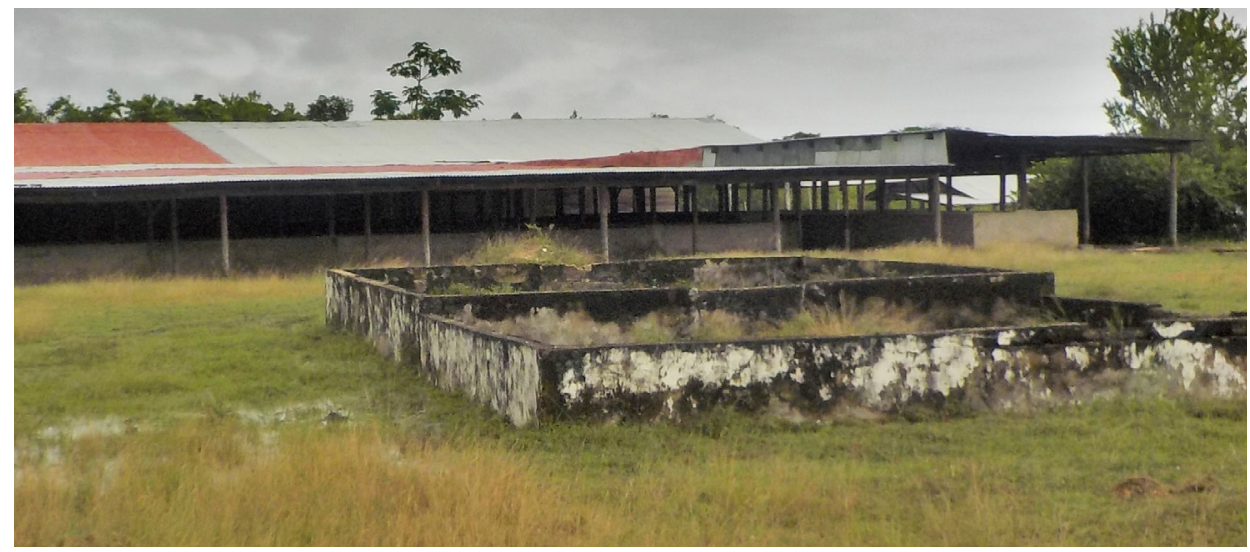

Fuente: Autor, Tuapi, 2017.

Figura 3: Medusa bola de cañon (Stomolophus meleagris).

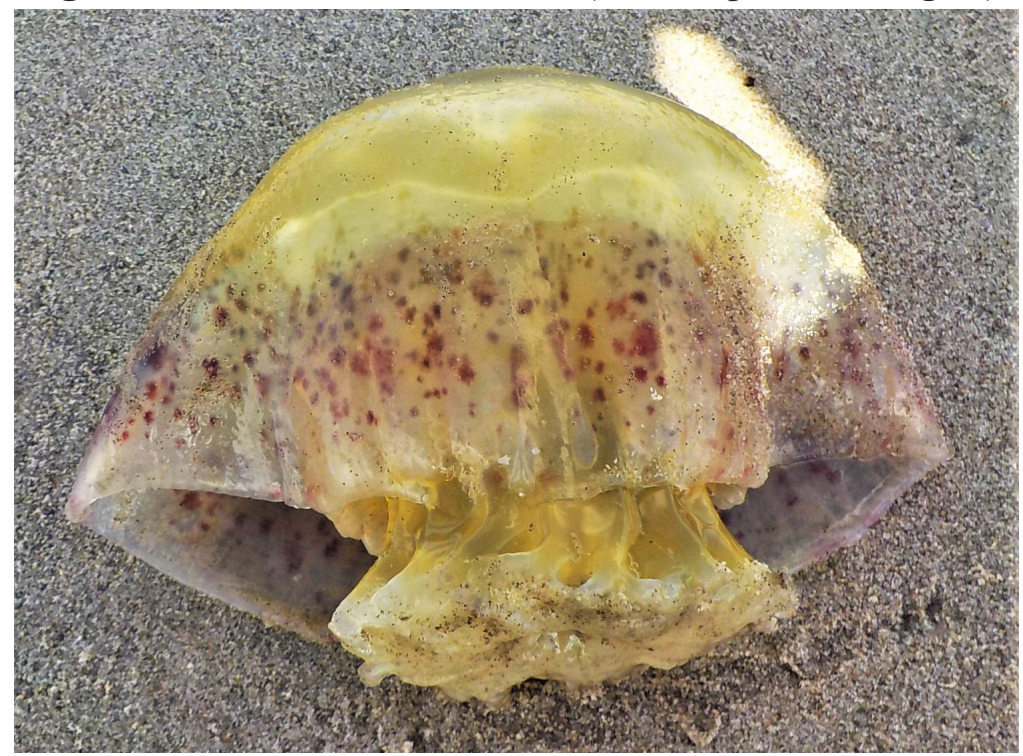

Fuente: Autor, Tuapi, 2017. 
En 2008, Moon negoció con INPESCA para iniciar una pesquería de medusas en la Costa. La medusa no tenía antecedentes de explotación en Nicaragua. No existía conocimiento institucional sobre la pesca de medusas e INPESCA no tenía idea de cómo regularlas. INPECSA estaba bajo presión internamente y desde la Asamblea Nacional, sin embargo, para desarrollar nuevas opciones para los pescadores artesanales y los buzos de langosta en la Costa.

Para muchas comunidades en la Costa, la actividad principal de subsistencia es la pesca artesanal (Henríquez y Webster 2010; González et al., 2011; Mendoza-Lewis et al., 2012) En 2015, el 71 por ciento de los mariscos desembarcados en la Costa fue capturado por pescadores artesanales (INPESCA 2016). Los pescadores, sin embargo, operan con márgenes estrechos y a menudo luchan para cubrir sus gastos. La sobreexplotación, el aumento de la competencia y la devaluación anual de la moneda, ha ocasionado que los pescadores y sus familias sufran cada año menores ingresos y aumenten los gastos (Henríquez y Webster 2010; González et al., 2011).

En, Estrategia para el Desarrollo Sostenible de la Pesca Artesanal, La Seguridad Alimentaria y la Reducción de la Pobreza de las Familias Viculadas 2008-2015, INPESCA admitió haber descuidado la pesca artesanal a pesar de la importancia de la actividad para las economías locales, especialmente en la Costa (INPESCA 2008). El objetivo para el período 2008-2015 era asumir un papel más activo con la pesca artesanal, incluida la identificación y el desarrollo de especies comercialmente viables no explotadas anteriormente (INPESCA 2008, 11, 30).

La agencia también se vio presionada para desarrollar alternativas para los buzos de langosta. En 2015, la langosta representó el 65 por ciento de las exportaciones de mariscos de la Costa por valor. La langosta, sin embargo, ha sido sobreexplotada en Nicaragua. González et al. (2014) estimaron que la cosecha de $2001 \mathrm{de} 1,890 \mathrm{mT}$ fue el 122 por ciento del rendimiento máximo sostenible; en 2015, la cosecha fue de 2,500 mT (INPESCA 2016). Tradicionalmente, la langosta fue capturada por buceo libre en aguas poco profundas. Mientras que muchos pescadores se han pasado a la pesca con trampas, los 2-3 miles buceadores restantes que aún trabajan se ven obligados a bucear con mayor frecuencia, a mayor profundidad y durante períodos más largos, utilizando equipo de buceo o compresores de aire (Farell 2010; INPESCA 2011; Wolff 2012; Acosta 2014). Esto y 
Matthew L. Fahrenbruch. Pesquerías de medusa en la Costa Miskitu: desafíos para la gobernanza comunal después de la Ley 445 de Nicaragua

la falta de capacitación y equipamiento adecuado han resultado en una epidemia de muertes y discapacidades relacionadas con la descompresión y demandas para prohibir la actividad (Farell 2010, INPESCA 2011, Wolff 2012, Acosta 2014).

En 2008, la Asamblea Nacional aprobó la Ley 613: Ley de Protección y Seguridad para las Personas Dedicadas a la Actividad de Buceo. La ley exigía la prohibición del buceo comercial por el 2011 y le encargó al INPESCA el desarrollo de alternativas para los buzos de langosta y los trabajadores de apoyo (ANN 2008). En 2009, Nicaragua firmó junto con otras naciones centroamericanas el acuerdo regional, OSP-02-2009 - Ordenamiento Regional de la Pesquería de la Langosta del Caribe, con el objetivo de eliminar el buceo de langosta en toda América Central (OSPESCA y OIRSA 2009).

Cuando Moon acercó a INPESCA en 2008, INPESCA vio a las medusas como una oportunidad para desarrollar un recurso previamente no explotado y al hacerlo proporcionar oportunidades para pescadores y buzos y también para mujeres y no pescadores en las plantas. En, el Plan de Reconversión Laboral Técnica y Ocupacional para los Pescadores que Utilizan la Tecnología del Buceo en la Pesca de Langosta, INPESCA destacó la pesca de medusas como una alternativa viable para los buzos y los trabajadores de apoyo (INPESCA 2011).

Desafortunadamente, a pesar de la buena producción que en ocasiones excedió la capacidad de las plantas, un rebote en la producción en el Golfo de California y una calidad de producto inaceptable hizo que Moon perdiera interés y abandonara la planta de Tuapi a fines de 2008 (La Prensa 2008; Brotz 2017 , Fahrenbruch 2018). Aproximadamente 204 mT de medusa fueron desembarcados ese año (INPESCA 2016).

Entre 2009 y 2012 no se desembarcó ninguna medusa en Nicaragua (INPESCA 2016). Sin embargo, entre 2013 y 2015, la industria volvió y se expandió rápidamente a nivel de producción industrial, a pesar de la falta de regulación. En 2013, se construyeron tres plantas adicionales; uno al sur de Tuapi y uno en Haulover y El Bluff. En 2013, los desembarques aumentaron a $635 \mathrm{mT}-211$ por ciento más que en 2008. En 2014, los desembarques aumentaron nuevamente 467 por ciento a más de 3.600 mT (INPESCA 2016). La planta original en Tuapi duplicó su tamaño y se construyeron dos plantas adicionales en Puerto Cabezas y Bismuhna. 
En 2015, se construyeron cuatro plantas adicionales en la Costa en Tuapi, Krukira, Awastara y Monkey Point.

En Tuapi, la planta operaba las 24 horas del día en algunos puntos y los barcos estaban alineados un kilómetro río abajo esperando para descargar. En su punto pico del 2014, se registraron más de 200 embarcaciones para entregar medusas a la planta original de Tuapi, que entonces era propiedad de la compañía internacional de mariscos, Mariscos Central y América Sur S.A. (MCASSA). El final, sin embargo, llegó rápidamente a fines de 2015.

$\mathrm{Al}$ igual que las pesquerías de medusas en todo el mundo, la pesquería en la Costa se basó en la ocurrencia natural de las floraciones de medusas estacionales (de octubre a marzo) (Kingsford et al., 2000). Durante estas floraciones, millones de medusas se congregarían por la superficie cerca de la costa donde los pescadores podrían sacarlas fácilmente con redes de cuchara. A finales de 2015, sin embargo, las floraciones de las medusas no se materializaron. Ese año, los desembarques cayeron 87 por ciento a $475 \mathrm{mT}$, y desde entonces no ha habido producción (INPESCA 2016).

\section{Desafíos para la gobernabilidad local}

\section{Desarrollo Centralizado}

El desarrollo de la industria de las medusas, al igual que las industrias previas, fue manejado desde arriba, impulsado por los intereses comerciales internacionales y los objetivos de desarrollo nacional. Moon International se acercó a INPESCA y trabajó con INPESCA en el desarrollo de la pesquería y las comunidades fueron consultadas al final del proceso, cuando las empresas necesitaban obtener su consentimiento. De acuerdo con los administradores anteriores de las plantas en Tuapi y Bismuhna, las compañías debían gastar miles de dólares para obtener permisos de INPESCA y otros funcionarios antes de que pudieran acercarse a comunidades potenciales.

La comunidad de Krukira, es una excepción. Durante una entrevista con el Sindico ${ }^{6}$ en abril de 2018, relataron que Krukira había suministrado muchos trabajadores y pescadores a la pesquería Tuapi. A través de esta experiencia, se familiarizaron con la industria y quisieron una planta para

6 El Sindico en una autoridad comunal encargada de la gestión de tierras y recursos. 
Matthew L. Fahrenbruch. Pesquerías de medusa en la Costa Miskitu: desafíos para la gobernanza comunal después de la Ley 445 de Nicaragua

Krukira. En 2014, los representantes de la comunidad se acercaron a las autoridades de la planta de Tuapi y propusieron que construyeran una planta en Krukira. Las autoridades declinaron pero los ayudaron a encontrar un inversionista para construir una planta. Si bien la planta solo funcionó durante unos meses en 2015, sirve como un ejemplo de cómo, a pesar del desarrollo centralizado de la industria, los residentes locales aprendieron y pudieron ejercer con éxito su autodeterminación para construir una planta en su comunidad.

Muchos líderes locales también expresaron su preocupación por el control de los ingresos obtenidos de la explotación de medusas. La Ley 445 prohíbe la venta de tierras comunales; por lo tanto, las plantas de medusas alquilaron sus tierras a las comunidades de acogida. Este fue el único ingreso recibido directamente por los gobiernos comunales y solo agregó un par de miles de dólares al año. Los ingresos de los permisos reales, por la Ley 445, son recaudados por el Gobierno Central a través del Ministerio de Finanzas y Crédito Público. La ley establece que los fondos se dividirán en un $25 \%$ cada uno entre la comunidad de acogida, el municipio y los gobiernos regionales y central, y se utilizarán para el beneficio directo de la comunidad de acogida (ANN 2003, Art. 34). Desafortunadamente, las comunidades no tenían un sistema de contabilidad para las medusas $\mathrm{u}$ otros recursos que salgan, por lo tanto, no tienen idea de si lo que están recibiendo es justo, si reciben algo en absoluto.

\section{Dominación extranjera de los mercados}

Otro desafío para la gobernabilidad local fue la dominación extranjera de la pesquería de medusas y la escasez de información a todos los niveles en Nicaragua sobre las pesquerías y los mercados de medusas. Según lo dispuesto por la Ley 445, se exigió a PBSA y a las empresas subsiguientes que obtuvieran el consentimiento de sus comunidades anfitrionas. Como resultado, las compañías negociaron directamente con las comunidades locales. Durante estas negociaciones, los representantes de la compañía presentarían sus proyectos en asambleas comunales, a menudo en español o en inglés, utilizando traductores locales de Miskitu cuando fuera necesario. Si la asamblea aprobaba el proyecto, las partes negociarían las rentas de la tierra y, en algunos casos, los precios de los muelles y el pago de la mano de obra. 
Los funcionarios de SERENA, INPESCA y otras agencias, en la mayoría de los casos, asistieron a las reuniones, sin embargo, no pudieron proporcionar información sobre pesquerías de medusas o mercados que las comunidades necesitaban para llevar a cabo negociaciones informadas. Las autoridades locales no tenían idea de cuál era el mercado para las medusas en el extranjero y si estaban negociando un precio justo. Tampoco tenían idea de cuál sería el impacto en las otras pesquerías de las que dependían.

En el caso de Bismuhna, ningún funcionario del gobierno se presentó a las reuniones y la compañía esencialmente dictó los términos y adoptó un enfoque de "tómalo o déjalo" (Bismuhna Sesión de Escucha, 18 de marzo de 2018). Las medusas no tenían historia de explotación en la Costa y ningun mercado local. Si los pescadores o los trabajadores querían participar, tendrían que vender sus medusas y mano de obra a las plantas. Si la planta se cerraba o sobrepasaba su capacidad, el mercado desaparecía y las medusas eran descamadas.

Un ejemplo de esto es la falla de la planta PBSA/MCASSA en Tuapi en 2017. MCASSA se hizo cargo de la planta Tuapi en 2014 de otra empresa de mariscos que la reabrió en 2013. Tras una buena producción en 2014, MCASSA firmó un contrato de arrendamiento de cinco años con Tuapi en 2015. MCASSA pagó $\$ 150$ al mes entre 2015 y 2017 a pesar de no haber procesado medusas. Sin embargo, el 22 de noviembre de 2017, MCASSA rompió su contrato con Tuapi y la comunidad asumió el control de la planta. Esto desencadenó dos días de debate en la comunidad sobre qué hacer con la nueva propiedad comunal.

Algunos querían publicitar la planta para ver si podían atraer a una compañía diferente. La mayoría, sin embargo, querían des-construir la planta y dividir los materiales entre los miembros de la comunidad; ellos veían los materiales de la planta, que podían usarse para reparar o aumentar sus casas, o venderse por dinero, como un valor más concreto para el comunidad que una planta de medusas que había estado inactiva durante tres años (Figura 4). La comunidad no tenía idea de si las medusas prosperarían en el futuro o nunca más y la compañía que los había conectado a la economía global había desaparecido. Al igual que con las economías de recursos mundiales pasadas que tocaron la costa, la comunidad se quedó con poco desarrollo, pero algunos desechos de madera, paneles de techado y alrededor de 2.000 bolsas de sal industrial y alambre. 
Matthew L. Fahrenbruch. Pesquerías de medusa en la Costa Miskitu: desafíos para la gobernanza comunal después de la Ley 445 de Nicaragua

Figura 4: Deconstrucción de la planta in Tuapi en noviembre de 2017.

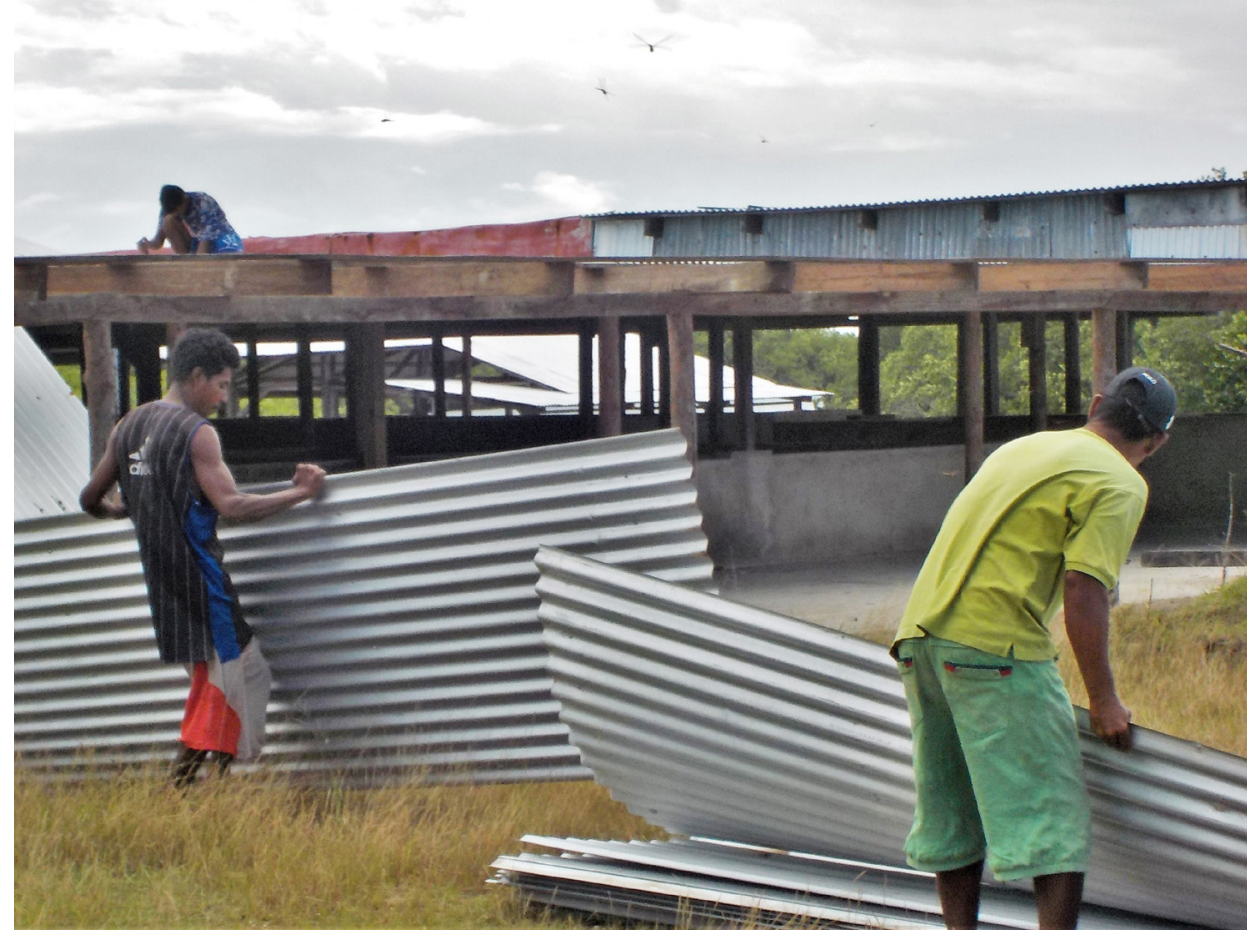

Fuente: Autor, Tuapi, 2017

\section{Conclusión}

La gobernanza de recursos en la Costa Miskitu se ha caracterizado históricamente por relaciones de poder asimétricas definidas por la dominación extranjera de los mercados, un fuerte control central y la marginación de los derechos territoriales y de los recursos indígenas y afrodescendientes. La esperanza de los grupos indígenas y afrodescendientes en la Costa era que la Ley 445 movería a la costa hacia un proceso de des-marginación a través del empoderamiento local.

El objetivo de este documento fue analizar el auge y caída de la pesquería de medusas en la Costa para resaltar algunos de los principales desafíos que aún enfrenta la gobernanza de los recursos comunales. Se discutieron tres desafíos principales. Primero, mientras que la Ley 445 garantiza a las comunidades indígenas y afrodescendientes el "pleno reconocimiento de los derechos de propiedad de propiedad comunal, el uso, 
administración y gestión de tierras tradicionales y sus recursos naturales ..." y establece un régimen de gobernanza multinivel que otorga el derecho de aprobar o rechazar concesiones en colaboración con los gobiernos regionales y centrales (ANN 2003, Arts. 2, 10, 12, 27), la ley ha sido desigual en su aplicación y no está claro para muchos donde estan trazadas las líneas de autoridad (Larson y Lewis 2012; Sylvander 2018). El desarrollo de la industria de las medusas siguió un modelo centralizado similar a las economías de extracción de recursos anteriores-impulsadas por los intereses comerciales internacionales y los objetivos de desarrollo nacional.

En segundo lugar, las comunidades carecen de control sobre los ingresos obtenidos a través de la explotación de medusas y otros recursos. Mediante la Ley 445, el gobierno central recauda, distribuye y supervisa los ingresos. A pesar del mandato de que los fondos se utilicen para beneficiar directamente a las comunidades de acogida, las comunidades de acogida no tienen sistemas de contabilidad para rastrear los recursos que salen sus territorios y no tienen idea de si lo que reciben, si reciben algo, es justo.

Por último, como en las economías de recursos anteriores, la pesquería de medusas estaba controlada por extranjeros. No había mercado local para las medusas y las medusas solo tenían valor cuando las plantas les daban valor. Los gobiernos comunales recibieron poca información sobre las pesquerías o mercados de medusas y se vieron en clara desventaja cuando negociaban con las compañías. Las medusas eran un recurso no tradicional y continua escasez de información en todos los niveles al respecto.

Los recursos no tradicionales son engañosos y requieren inversión para comprenderlos a ellos y sus mercados. A pesar de las preocupaciones presupuestarias, las agencias nacionales están mejor posicionadas para generar este conocimiento. Necesitan hacerlo y compartir esta información a través del régimen multinivel. Las comunidades locales necesitan una mayor inclusión en los planes de desarrollo, no solo en la consulta. Ejemplos como Krukira demuestran que las comunidades están interesadas y pueden tomar la iniciativa cuando están informadas, pero necesitan estar informadas.

La situación actual con la pesquería es mixta. A partir de abril de 2018, varias plantas, incluidas las de Krukira, Bilwi, Haulover y la segunda construida cerca de Tuapi, todavía estaban pagando el alquiler a sus comunidades de acogida e intentaban diversificar los tipos de productos que podían procesar. Al menos dos de las plantas, la tercera 
construida cerca de Tuapi y la planta en Awastara se construyeron a fines de 2015 y nunca operaron.

Para complicar aún más las cosas, el levantamiento del movimiento del 19 de abril en el Pacífico ha traído una gran inseguridad al país a medida que el movimiento popular busca desalojar al dictador Sandinista Daniel Ortega. Si el movimiento tiene éxito, es probable que también tenga un gran impacto en la gobernanza a todos los niveles en Nicaragua, incluida la gobernanza de los recursos en la Costa Miskitu. Esta inseguridad ya ha tenido un efecto de enfriamiento en el turismo y la inversión en el país y, al final, puede tener un mayor impacto en el futuro de la pesquería de medusas, más que las propias medusas.

\section{Expresiones de gratitud}

Gracias a la gente de la Costa que compartieron conmigo sus experiencias con la pesquería de medusas. Gracias a Keith Narváez y Lenin Green de la Universidad de las Regiones Autónomas de la Costa Caribe de Nicaragua por su asistencia logística.

También gracias a mi asesor de doctorado, el Dr. Peter Herlihy, quien me apoyó con una asistencia de investigador graduado de 2014 a 2017 en su Proyecto, Centroamérica Indígena, financiado por una beca de la Iniciativa de Investigación Minerva (W911NF1310281). Gracias también a la Dra. Aida Ramos por su ayuda para revisar este artículo.

Esta investigación se llevó a cabo con e una Beca Fulbright de los Estados Unidos, una Beca Stansifer y una Beca Oppenheimer del Centro de Estudios Latinoamericanos y del Caribe de la Universidad de Kansas, y una Beca de Investigación de Campo Tinker Foundation. Este estudio fue aprobado por la Junta de Revisión Interna de la Universidad de Kansas. 


\section{Referencias}

Acosta, M. L. (2014). Lobster Divers in Nicaragua's Atlantic Coast. Manuscript, Center for Legal Assistance to Indigenous Peoples (CALPI). Obtenido Junio 10, 2018, de https://www.calpi-nicaragua.org/ lobster-divers-in-nicaraguas-atlantic-coast/.

ANN (Asamblea Nacional de Nicaragua). (2003). Ley No. 445: Ley del Regimen de Propiedad Comunal de los Pueblos Indígenas y Comunidades Etnicas de las Regiones Autonomas de la Costa Atlántica de Nicaragua y de los Rios Bocay, Coco, Indio Y Maiz. La Gaceta: Diario Oficial de Nicaragua, 16: 313-321.

ANN (Asamblea Nacional de Nicaragua). (2008). Ley No. 613: Ley de Protección y Seguridad a las Personas Dedicadas a la Actividad de Buceo. La Gaceta: Diario Oficial de Nicaragua, 12: 382-415.

Baracco, L. (2011). From Developmentalism to Autonomy: The Sandinista Revolution and the Atlantic Coast of Nicaragua. En: National Integration and Contested Autonomy: The Caribbean Coast of Nicaragua. L. Baracco (Ed.) pp. 117-146. Algora Publishing: New York.

Brotz, L. (2017). Jellyfish Fisheries of the World. Unpublished Ph.D. Dissertation, University of British Columbia.

Brotz, L., A. Schiariti, J. López-Martínez, et al. (2017). Jellyfish fisheries in the Americas: origin, state of the art, and perspectives on new fishing grounds. Reviews in Fish Biology and Fisheries, 27(1): 1-29.

Fahrenbruch, M.L. (2017). "Cucharear dinero del mar”: La producción de medusas en La Mosquitia. Wani Revista del Caribe Nicaragüense, 72:31-38.

Fahrenbruch, M.L. (2018). 'Like scooping money out of the sea': Chinese luxury seafood consumption, roving bandits, and the boom-and-bust jellyfish fishery on the Miskitu Coast of Nicaragua. Journal of Latin American Geography, 17(2): En Prensa.

Farell, N. (2010). Nicaragua before now: factory work, farming, and fishing in a low-wage global economy. Albuquerque, NM: University of New Mexico Press.

González, M. (2011). To Make a Fishing Life: Community Empowerment in Small-scale Fisheries in the Pearl Lagoon, Nicaragua. En: Poverty Mosaics: Realities and prospects in Small-Scale Fisheries, S. Jentoft and A. Eide (Eds.) pp. 275-308. London: Springer. 
Matthew L. Fahrenbruch. Pesquerías de medusa en la Costa Miskitu: desafíos para la gobernanza comunal después de la Ley 445 de Nicaragua

González, M, E. Jackson, and Y. Zapata. (2014). Análisis de la economía y los sistemas políticos de la costa caribe. Wani Revista del Caribe Nicaragüense, 31:6-29.

Hale, C.R. (1994). Resistance and Contradiction: Miskitu Indians and the Nicaraguan State, 1894-1987. Stanford University Press: Stanford, CA.

Henriquez, U., M. T., and Y. B. Webster D. (2010). Impacto socio-económico de la Pesca Artesanal en la comunidad de Karata, Enero-Julio, 2009. Monografía. URACCAN: Puerto Cabezas - Bilwi.

Helms, M. (1971). Asang: Adaptions to Culture Contact in a Miskito Community. University of Florida Press: Gainesville, FL.

Hsieh, Y-H. P., F-M. Leong, and J. Rudloe. (2001). Jellyfish as Food. Hydrobiologia, 451:11-17.

INPESCA (Instituto Nicaraguense de la Pesca y Acuicultura). (2008). Estrategia para el Desarrollo sostenible de la Pesca Artesanal, La Seguridad Alimentaria y la Reducción de la Pobreza de las Familias Vinculadas 2008-2015. Managua: INPESCA.

INPESCA (Instituto Nicaraguense de la Pesca y Acuicultura). (2011). Plan de Reconversión Laboral Técnica y Ocupacional para los Pescadores que Utilizan la Técnica del Buceo en la Pesca de Langosta. Managua: INPESCA.

INPESCA (Instituto Nicaraguense de la Pesca y Acuicultura). (2016). Anuario Pesquero y Acuicola, 2015. Oficina de Estadisticas: Managua, Nicaragua. Obtenido Junio 3, 2018 de http://www.inpesca.gob.ni/ images/DocumentosSubidos2016/ANUARIO\%20PESQUERO $\% 20$ Y\%20ACUICOLA\%20DE\%20NICARAGUA\%202015.pdf

Kingsford, M.J. K.A. Pitt, and B.M. Gillanders. (2000). Management of Jellyfish Fisheries, with Special Reference to the Order Rhizostomeae. Oceanography and Marine Biology, 38: 85-156.

La Prensa. (2008). Aguamala Para Exportación. La Prensa, October 13. Obtenido

Junio 3, 2018 de https://www.laprensa.com.ni/2008/10/13/ departamentales/1344220-aguamala-para-exportacion.

Larson, A.M. and J. Lewis-M. (2012). Decentralization and devolution in Nicaragua's North Atlantic Autonomous Region: natural resources and indigenous peoples' rights. International Journal of the Commons, 6(2): 179-199. 
Mendoza-Lewis, J., M. Dalvez, and K. Narváez. (2012). Estudio de caso del territorio indígena Tawira. En: Territorialidad y Gobernanza: Tejiendo retos en los territorios indígenas de RAAN, Nicaragua. A. Larson and Y. Fenanda-Soto (Eds.) pp. 177-222. Managua: Instituto de investigación y Desarrollo Nitlapan de la Universidad Centroamericana.

Nietschmann, B. (1973). Between Land and Water: The Subsistence Ecology of the Miskito Indians, Eastern Nicaragua. Seminar Press: New York.

Nietschmann, B. (1979). Chapter 6: When the Turtle Collapses, the World Ends. En: Caribbean Edge: The Coming of Modern Times to Isolated Peoples and Wildlife. The Bobbs-Merrill Co, Inc.: New York.

Nietschmann, B. (1997). Protecting Indigenous Coral Reefs and Sea Territories, Miskito Coast, RAAN, Nicaragua. En: Conservation through Cultural Survival: Indigenous Peoples and Protected Areas. S. Stevens (Ed.) pp. 193-224. Washington, D. C.: Island Press.

Offen, K. H. (2004). The Geographical Imagination, Resource Economies and Nicaraguan Incorporation of the Mosquitia, 1839-1909. En: Territories, Commodities, and Knowledges: Latin America Environmental Histories in the Ninetieth and Twentieth Centuries. C. Brandnstrom (Ed.) pp. 50-89. New York: Institute of Latin American Studies.

OSPESCA and OIRSA (la Organización del Sector Pesquero y Acuícola del Istmo Centroamericano and el Organismo Internacional Regional de Sanidad Agropecuaria. (2009). Reglamento OSP-02-09 para el Ordenamiento Regional de la Pesquería de la Langosta del Caribe (Panulirus argus). El Salvador: OSPESCA and OIRSA.

Pineda, B. (2006). Shipwrecked Identities: Navigating race on Nicaragua's Mosquito Coast. New Jersey: Rutgers University Press.

Rudloe, J. (1995). Harvesting \& Processing of Florida Cannonball Jellyfish. Panacea, Fl: Gulf Specimen Marine Laboratories.

Sylvander, N. (2018). Saneamiento Territorial in Nicaragua, and the Prospects for Resolving Indigenous-Mestizo Land Conflicts. Journal of Latin American Geography, 17(1):166-194.

Wolff, J. (2012). My Village, My Lobster. Nomading Films. 